\title{
Surface Treated Catheters for Vascular Access-Useful?
}

\author{
Rolf Bambauer $^{1 *}$, Ralf Schiel ${ }^{2}$, Carolin Bambauer ${ }^{3}$, Reinhard Latza ${ }^{4}$ \\ ${ }^{1}$ Institute for Blood Purification, Homburg, Germany \\ ${ }^{2}$ Inselklinik Heringsdorf GmbH, Seeheilbad Heringsdorf, Germany \\ ${ }^{3}$ Main Hospital Darmstadt, Darmstadt, Germany \\ ${ }^{4}$ Laboratorium of Medicine, St. Ingbert, Germany \\ Email: *rolf.bambauer@t-online.de
}

Received July 1, 2013; revised July 29, 2013; accepted August 11, 2013

Copyright (C) 2013 Rolf Bambauer et al. This is an open access article distributed under the Creative Commons Attribution License, which permits unrestricted use, distribution, and reproduction in any medium, provided the original work is properly cited.

\begin{abstract}
Background: Catheter-related infections (CRI), thrombosis, and stenosis are among the most frequent complications associated with catheters which are inserted in vessels as vascular access. These problems are usually related to the handling of the staff, the catheter materials, and the surface properties of the catheter. To mitigate such complications surface treatment process of the outer surface, such as ion beam assisted deposition is investigated in a retrospective study from 1992 to 2007, to prove if the surface treatment of the catheters is a sufficient solution. Methods: This study (1992-2007) evaluated silver coated and non-coated implanted large-bore catheters used for extracorporeal detoxification. In 159 patients, 54 patients received a silver coated catheter (Spi-Argent, Spire Corporation, Bedford, MA, USA) and 105 patients, an untreated catheter served as controls. The catheters were inserted into the internal jugular or subclavian veins. After removal, the catheters were cultured for bacterial colonization using standard microbiologic assays. They were also examined using a scanning electron microscope (SEM). Results: The silver coated catheters showed a tendency towards longer in situ time. The microbiologic examinations of the catheter tips were in both catheter types high positive, but not significant. Conclusion: The silver coated catheters showed no significant reduction in infection rate by evaluation of all collected data in this retrospective study. There was no association between both catheters in significantly reducing patient discomfort. Other surface treatments which include the outer and inner surface are necessary. New developed catheter materials such as the microdomain structured inner and outer surface, as an example, are considered more biocompatible because they mimic the structure of natural biological surface.
\end{abstract}

Keywords: Surface Treated Large Bore Catheters; Hemodialysis; Apheresis; Ion Been Assisted Deposition (IBAD); Microdomain-Structered Surface (PUR-SMA Coated Catheter)

\section{Introduction}

Since the first introduction of large-bore catheters for acute hemodialysis in 1961 by Shaldon et al. [1], many problems with handling, materials, and contamination of these catheters have arisen. Nevertheless, vascular catheters have become essential tools for management of hospitalized or chronically ill patients requiring intensive medical treatments [2]. The increased insertion of such devices has been accompanied by a corresponding increase in complications, such as bloodstream infection and thrombosis [3]. Infections are particular concern, because they can appear at any time, even years after implantation, and are not material dependent. They are usually attributed to microbial colonization of the skin or handling of the catheters by attending staff. Complication

"Corresponding author. rates due to venous catheter-relates infection are reported to range from 34 to 40 percent [4,5]. Despite recent technical innovations in hemodialysis, problems related to temporary or permanent vascular access have found no satisfactory solutions. Temporary vascular access is particularly problematic.

Catheterization of the femoral vessels produces more complications than the catheterization of the superior vena cava (SVC). Cannulation of the SVC versus the subclavian vein is difficult to implement and involves a high complication rate [6]. Using the infraclavicular catheterization technique is often difficult to push the large-bore catheter under the clavicle. Because of the anatomical position of the subclavian vein, perforation is more likely with a rigid, large-bore catheter, apart from the danger of causing a pneumothorax or a hemathorax [7-10]. 
Therefore, Bambauer et al. have introduced the insertion of large-bore catheters in the superior vena cava rather than in the internal jugular or the subclavian veins $[11,12]$. Dialysis catheters are used for vascular access in $65 \%$ of incident hemodialysis (HD) patients, and in $25 \%$ of the prevalent HD populations [13]. Today, the first choice of vascular access is the vena cava superior over the internal jugular vein.

Catheter-related bacteremia is a major cause of morbidity among hemodialysis patients. Treatment with systemic antibiotics alone without removal of the catheter fails to definitely eradicate the infection in most patients [14]. Catheter-related bacteremia must be managed by either catheter removal with delayed placement of a new catheter or management of the infected catheter with a new catheter over a guide-wire and additional systemic antibiotic therapy. These catheter-related complications are contributing factors to increasing cost of medical care. They are responsible for patient readmissions and longer hospital stays as well as patient discomfort, morbidity, and occasional mortality.

The source of catheter-related bacteremia is in most patients a bacterial biofilm that forms in the catheter lumen or on the outer surface. This biofilm, most consisting of staphylococcus aureus, cannot be destroyed or eliminated by a systemic antibiotic therapy because of antimicrobial resistance [15]. Bacteriae could most of the time colonize rough surfaces [16]. The combination of rough surfaces and protein deposits should be an ideal situation for the colonization of bacteria. The bacteria could produce and become covered with a slime layer, in which case antibiotic drugs have no influence on the bacteria. The bacteria under the slime layer use the organic substances of the catheter material for their metabolism. The toxins of the bacteria can penetrate the slime layer and enter the patient blood provoking a catheter infection [16]. Biofilm is a microbial derived sessile community characterized by cells that are irreversibly attached to a substratum or interface to each other, embedded in a matrix of extracellular polymeric substances that have produced [17]. Such a biofilm can be the origin of fibrin sheath formations leading to catheter dysfunction due to blood reducing and to blood disturbances. The therapy must be to remove the catheter immediately, or exchange it over a guide-wire with a new catheter and additional systemic antibiotic therapy.

Biocompatibility of synthetic materials is another major problem. The interaction of blood with a synthetic surface causes coagulation and activation of the complement system. This can lead to the adsorption of various proteins and the formation of a layer of protein on the synthetic surface. Thrombocytes, other cells and bacteria adhere to this layer of protein so that thrombi may form which can lead to blood flow disturbances and catheter dysfunction [18].

To influence catheter-related bacteremia different new developments are available today, such as new catheter materials, coating of the catheter surface with antibioticheparin, or silver and silicone, cuffs on the outer surface, catheter for tunnelling, installation of an antibiotic-anticoagulant lock into the catheter lumen after the HD, etc. $[14,19,20]$. The first results with available catheters which coated on the outer surface with silver or silicone were encouraged [18,21].

In a retrospective study from 1992 to 2007, outer surface treated catheters with silver versus untreated catheter in 159 patients, who needed a large-bore catheter, were investigated. The results of a preliminary study from 2001, which showed $75 \%$ decline in the infection rate with the surface treated catheters cannot be confirmed with the present study. One reason may be that on the surface treated catheters only the outer surface was coated with silver and the possibility of contamination by the handling during the extracorporeal treatments.

Therefore, new materials and surface treatment technologies are needed to save health care costs for hemodialysis catheters, to reduce infection rates and thrombus formations and to help improve the patients' outcome. The handling of the catheter by the attending staff must be improved and done after the guidelines of different medical communities [22]. In this study, the authors try to give an overview of the surface treated catheters and show a new technique of microdomain-structured surface catheters (PUR-SMA coated catheters, (Gambro, Germany) as an example if these technologies are useful in reducing catheter-related infections and thrombogenicities.

\section{Catheter and Material}

All available single-, double-, or triple-lumen catheters have some deficiencies depending on the material. Not all catheters are radiopaque. No problem is experienced with the polyurethane catheters after the incorporation of contrast media; however, the latter material may affect catheter durability when using Teflon. This problem was overcome by making a thicker catheter wall, but this caused endothelial irritation and early thrombus formation [23]. Catheters providing radio contrast are not absolutely necessary however, because their position can be controlled more simply and gently with an intra-atrial electrocardiogram (ia-ECG) lead [24]. The three most important criteria of any catheter material are a good tolerance, a low thrombogenicity, and a low infection rate.

Rarely do the material properties perfectly match every requirement in a given application and biomaterials are no exception. Therefore, it often becomes necessary to strike a compromise so that a material has acceptable 
properties. For example, in a product such a hemodialysis catheter, which demands both good flexibility and low surface friction, the best candidate may be a slippery, less flexible material rather than a more supple one with unacceptable high friction [25].

The importance of surface-engineered biomaterials has been recognized by major medical device companies, because surface modification processes can reduces the rate of infection, thrombogenicity, and other catheter-related complications without adversely affecting the basic design function of catheters.

Although the field of surface-engineered biomaterials is still essentially in its infancy, the range of services currently offered by surface treated vendors is varied and continually expanding. Surface modification processes can reduces the rate of infection, thrombogenicity, and other catheter-related complications without adversely affecting the basic design function of catheters. Examples include conventional coating process such as depending and spraying: vacuum-deposition techniques (e.g., sputtering), and surface modification approaches such as diffusion (e.g., nitriding, carburizing), laser and plasma processes, chemical plating, grafting or bonding, and bombardment with energetic particles (as in plasma immersion or ion implantation). Of the available techniques, those based on ionised particle bombardment have been particularly successful in biomaterial surface modification, primarily because they combine versartility and low-temperatures processing with superior control, reliability, and reproducibility $[25,26]$.

The ion beam-based technology used for the treatment of catheters covered herein is ion beam-assisted deposition (IBAD: Spi-Argent ${ }^{\mathbb{}}$, Spire corporation, Bedford, MA, USA) [21,25,27]. The process is typically performed at low temperature under high vacuum. The affected layer in the typical films deposited by the IBAD process, is in the order of $1 \mu \mathrm{m}$ or less vacuum- compatible catheter materials may, therefore, be treated without adversely affecting bulk mechanical properties. The IBAD is line-of-directly: however, parts with complicated geometries may be manipulated for uniform coverage of all surfaces [26].

Silver has been indicated as a good prospect for an infection-resistant coating material for catheters. The problem previously preventing the use of silver on catheters has been the inability to deposit adherent films of silver on flexible polymeric substances. The IBAD process permits the formation of silver coatings at a relatively low temperature with extremely good adhesion that prevents delamination of the film during extended exposure to bodily fluids. The IBAD silver-deposited film has a low coefficient of friction, is highly uniform, and has a cytotoxicity test and the USP Systemic Injection Test. Excellent results were obtained in both tests $[25,26$,
28,29].

Another possibility shows the new developed catheter material, the microdomain structured surface (PUR-SMA coated catheter, Gambro Germany) [23]. Microdomain surfaces are considered the most biocompatible because the mimic the structure of natural biological surfaces. Microdomain structures are used to match the multiple requirements for improved catheter surfaces, that is reduced thrombogenicity and improved antimicrobial properties. An SMA-modified polyurethane coating consists of hydrophobic and hydrophilic microdomain in range below $50 \mathrm{nmm}$. Up to 50 percent of the molecule is presented to the surface and creates microdomain structured surfaces. If the domains are below a critical dimension of approximately $100 \mathrm{nmm}$, theoretical considerations indicate that interaction with proteins, blood cells, or even bacteria will be unstable and therefore not occur as frequently as on non-microdomain structured surfaces.

\section{Patients}

In the retrospective study of a single center from 1992 to 2007, all catheter data of all included patients were collected from the patients's charts. The inclusion criteria were patients $>18$ years of age who required a large-bore catheter (in-/outpatient), were free of bacteremia and provided informed consent. The exclusion criteria were a pregnant or lactating female, a hypersensitivity of silver and a bacteremia at the time of catheter insertion. An IRB approval was in 1992 not necessary [30]. After the patients had given their consent to this study, the physician chose the catheter which he inserted after a randomization of one surface treated catheter, and than two untreated catheters, and so on.

In the study a total of 159 patients (age $66.5 \pm 13.2$ years, female $\mathrm{n}=94(59 \%)$ ) are involved. Large-bore, single-lumen catheters were inserted percutaneously in the internal jugular or subclavian veins. The percutaneously catheterization was necessary in renal failure because of acute kidney injury (AKI) for hemodialysis due to cardio-vascular disease, postoperative AKI etc., and in end-stage renal disease (ESRD) because of clotting fistula, septicaemia, abscess and catheter thrombosis and faults in the catheter material $(n=138(86.8 \%))$. Further indications of catheterization were access problems in patients with familial hypercholesterolemia $(\mathrm{n}=12$ (7.5\%)) under LDL-apheresis treatment, different indications for plasmapheresis $(\mathrm{n}=7(4.4 \%))$ and in 2 patients with carcinoma $(\mathrm{n}=2)(1.3 \%))$.

In 54 patients $(34 \%)$ a catheter with silver coating on the outer surface (Spi-Argent ${ }^{\circledR}$, Spire, Bedford, MA, USA) was inserted and 105 patients $(66 \%)$ received untreated catheters after a randomization of one treated and two untreated catheters. Patients with untreated catheters were younger $(62.2 \pm 16.2$ versus $68.8 \pm 10.7, p=0.003)$ 
but there were no differences between the groups regarding gender distribution, diagnosis, or extracorporeal detoxification methods. Catheterization must always be done under aseptic conditions (wearing sterile gown each time, sterile gloves, mask etc). The patient should be correctly positioned, according to the vascular access point to be used and should be given adequate local anesthetic.

The catheters were placed by nephrologists after the Seldinger technique and/or under fluoroscopic guidance. Before percutaneous insertion each patient skin was disinfected using a consistent method, and a sterile skin smear was taken for microbiologic examination, and than the catheter was inserted. Before fixing the catheter with a suture, its position (particularly the catheter tip) should be checked with a normal radiological control and/or with an ia ECG [24]. Before and after the extracorporeal detoxification procedures, the staff worked under sterile conditions with disinfection and sterile gloves etc. In long-term catheters, a blood smear was taken every 4 weeks or earlier if an inflammation was seen on the insertion side of the catheter to screen for bacteria. Catheters were removed either when other vascular access routes became available or when serious infections developed, or if the catheter was not longer necessary.

Before catheter removal, a skin smear was taken. The catheters were then removed under sterile conditions, and the tip was examined bacteriologically. The remainder of the catheter was rinsed in physiological saline solution and fixed in a solution of phosphate buffer containing glutaraldehyde and formaldehyde for histological investigation.

\section{Statistical Analysis}

Statistical analysis was performed using the Statistical Package for Social Sciences (SPSS 13.0). All continuous data are presented as mean \pm standard deviation (SD) or if the data showed no normal distribution, as median and range. Dichtomous data were presented as a number (n) or in percent $(\%)$. Univariante, unadjusted analysis were performed with the independent samples t-test, chisquare test, Fisher's exact test for frequencies at or below 5 and the Wilcoxon's rank sum test. Pearson's correlation coefficient was calculated and multivaria's analysis was used to evaluate the presence of associated variables. Significance was defined at the 0.05 level.

\section{Results}

The median in situ period untreated and silver coated catheters were 138.9 (range, 1 - 1,845) and 115.0 (range, $4-1,348)$ days respectively $(p=0.653)$. Calculating the in situ times after classification for different age groups, it will be overt, that in patients older than 45 years in situ times were significantly longer $(p<0.01)$. Comparing the in situ times of untreated catheters after classification for in situ times, there was a tendency towards longer in situ times for the silver coated catheters. In the median catheters were used for 44 (range, 1 - 670) treatment sessions. Untreated catheters were used for 51 (range, 1 625 ) treatments, silver coated catheters for 39 (range, 1 $670, \mathrm{p}=0.849$ ) treatment sessions [30].

Performing microbiologic examinations of the catheter tips some differences were overt. Of the untreated catheter tips $55 \%$ cultured positive for bacteria. Of the cultures in patients with surface treated catheters $52 \%$ were positive, not significantly lower. Although untreated catheters showed a lower infection rate with Staphylococcus aureus, in treated catheters the infection rate with Staphylococcus epidermidis, pseudomonas, and others such as saphrophytes was not significantly lower (Table 1). A catheter thrombosis rate was not proved in the removed catheters.

Performing multivariante analysis there was a strong association between catheters' in situ period (R-square = $0.96)$, the number of treatment sessions $(\beta=0.97, \mathrm{p}<$ $0.001)$ and patients' age $(\beta=0.095, \mathrm{p}=0.002)$. There was no association between the in situ time and silver coated/untreated catheters, results of the bacteriological examination, and patients diagnosis outcome. Catheter malfunction or fibrin sheath formation as an outcome of both groups was not investigated.

The decrease of the infection rate in surface treated catheter in the preliminary study from 2001 cannot be seen in this presented study from 1992 to 2007. An explanation could be that all and more available data are now evaluated. The untreated catheters showed a higher positive culture for bacteria of $55 \%$ versus $52 \%$ to the surface treated catheters, but without significance. The procedure for both studies was the same.

The PUR-SMA coating prevents contact of blood components with barium sulphate, possibly leading to leaching as particles or dissolved in the surrounding media. The advantage of the PUR-SMA surface treatment is the coating of the inner and the outer surface in contrast to the ion beam-based surface treatment technologies in which can be treated only the outer surface of the catheters. The preliminary results with these PUR-SMA coated catheters showed a good biocompatibility without any blood deposits and a low thrombogenicity and coagulation activity. The microbiological results were low and of those from the Spi-Argent ${ }^{\circledR}$ catheters [30].

\section{Discussion}

Catheter-related infections are the most dangerous complications of large-bore catheter aside from accidential puncture of an artery. In addition to colonization, biocompatibility of a catheter material is an important con- 
Table 1. Microbiological examinations of 105 untreated and 54 surface treated catheters.

\begin{tabular}{|c|c|c|c|c|c|}
\hline Microorganism & Untreated (n) & $\%$ & Treated (n) & $\%$ & p-value \\
\hline Negative & 47 & 45 & 26 & 48 & n.s \\
\hline S. aureus & 31 & 29 & 21 & 38 & n.s \\
\hline S.epidermidis & 7 & 7 & 1 & 2 & n.s. \\
\hline Pseudomonas & 1 & 1 & 0 & 0 & n.s. \\
\hline Enterobacter & 1 & 1 & 1 & 2 & n.s. \\
\hline Others & 18 & 17 & 5 & 10 & n.s. \\
\hline
\end{tabular}

tributing factor to a successful clinical outcome, particularly in catheters that remain in situ for several weeks or months. Although improved since the use of centrally placed catheters, the incidence of catheter clotting was previously very high.

Infection rates range from 5\% to $30 \%$ and the most bacteria found is the Staphylococcus aureus. These rates do not depend on the route of vascular access [31]. Catheter-related Staphylococcus aureus bacteremiae are one of the main causes of morbidity and preventable cause of death in hemodialysis. Patients on dialysis are at a high risk of Staphylococcus aureus bacteremia and they have a four times higher mortality from central venous catheter-related Staphylococcus aureus bacteremia than other patients [15,32,33].

Recent data have suggested that methicillin-resistant $S$. aureus (MSRA) and vancomycin intermediate $S$. aureus (VISA) organisms may have increased [34]. One of the proposed mechanisms of vancomycin resistance is the bacterial cell wall thickening following vancomycin exposure [35]. Vancomycin's activity may be decreased due to the thickness of the bacterial cell, the results are MSRA and VISA [36].

To reduce infection rates and thrombogenicity, coated catheters and cuffs were investigated [37-40]. The clinical results of our preliminary investigations showed a significantly reduced infection rate in treated versus untreated catheters, a reduction of more than $75 \%$ [18]. With the silver surface treatment, a very smooth metallic surface was obtained which was responsible for a lower thrombogenicity rate. The activation of coagulation factors at the catheter surfaces, and the catheter thrombosis rate was not investigated. Silver ions are bactericidal, therefore, no bacteria growth is possible on the treated catheter surface. The positive association between the in situ time of the catheters and the patients' age may be because of an alteration of the immune system in elderly patients, especially in hemodialysis patients.

But in our retrospective study of all silver coated catheters no significantly reduction in infection rate, improvement, or life expectancy of silver coated versus untreated catheters, which were inserted during 1992 and 2007, was observed. One reason can be that with the
IBAD technology only the outer surface is coated with silver. The postulated penetration of silver ions from the outer to the inner surface cannot be shown with these results. The only outer surface treated surface catheters with silver have no advantage in point of view of reducing infection rate and improvement of patients versus the untreated catheters. The handling of the catheters under sterile conditions before, during and after the extracorporeal treatments probably cannot prevent the contamination with bacteria, especially the untreated inner side.

Based on these results, new materials must be developed, which should have better biocompatibility to reduce side effects so that they can be left in situ for a long time, because the part of dialysis patients with vascular problems is increasing in the last decade, and now about $30 \%$ of all hemodialysis patients [41], because the age of HD patients is permanent growing up. As the requirement for more and more artificial organs and/or organ replacement increases, especially in elderly patients, there will be a definite need for new materials with better biocompatibility and for suitable technologies to solve these infection, thrombosis and medical problems to reduce the costs and get better improvement of patients. A disadvantage of drugs such as antibiotics in the catheter surfaces or administration to patient or disinfection substances is that they can develop resistance by mutation or other mechanisms. Therefore the need of new surgical techniques and materials are necessary [42].

More new materials must be developed, which should have better biocompatibility to reduce side effects so that they can be left in situ for a long time, because the part of dialysis patients with vascular problems is increasing in the last decade. As the requirement for more and more artificial organs and/or organ replacement increases, there will a definite need for new materials with better biocompatibility and for suitable technologies to solve these infection, thrombosis and medical problems to reduce the costs and get a better improvement of patients. But it appears impossible to create a surface with an absolute "zero" adherence due to thermal-dynamical reasons and due to the fact that a modified material surface is in vivo rapidly covered by plasma and connective tissue proteins. 
Therefore other concepts of the prevention of implantassociated infections must involve the impregnation of the devices the inner and outer surface with antibiotics, antimicrobial substances and/or metals [43,44]. Another point is to understand the processes leading to the development of catheter-related bacteremia in order to can offer effective preventive and therapeutic possibilities [45] such as new polymer-antibiotic systems in inhibiting bacterial biofilm formation and in reducing neutrophil activation after surface contact on different biomaterials, thus reducing the risk for biomaterial-mediated inflammatory reactions [46-48], or the development of new biofilm to serve in a communication system termed quorum sensing [49], or molecules that inhibit quorum sensing signal generation among organism could block microbial biofilm formation [50].

These catheters related complications are contributing factors to the increasing cost of medical care. They are responsible for patient readmissions and longer hospital stays as well as patients discomfort, morbidity, and occasional mortality. Feldman et al. calculated in 1996 the costs of the morbidity due to catheter infections will soon exceed $\$ 1$ billion per year [51]. Therefore he demanded to reduce vascular access-related morbidity, that strategies must be developed not only to prevent and detect appropriately early synthetic vascular access dysfunction, but to better identify the patients in a whom radial arterio-venous fistula is a viable clinical option. The representative health care cost savings for hemodialysis catheters, given specific infection rates and potential infection rate reductions achieved by treated catheters [23].

The cost analysis was calculated using the literature and the available costs of different companies which distribute these catheters [52]. Potential health care cost reductions that could be achieved through the use of surface treated-catheters by an annual usage of 125,971 hemodialysis catheter devices and an infection rate of 5\% $20 \%$, savings per year of $\$ 17.7$ million, reduction about $40 \%$ [52]. Besides a high number of patients who die to CRI, the costs of these infections are increasingly steady. After Schwebel et al. the costs are \$2118/intensive care unit day, and after Pronovost et al. \$ 45,000 per each infection [53,54]. Tacconelli et al. estimated in 2009 the costs associated with CRI in four European countries (France, Germany, Italy, and UK) between $€ 35.9$ and $€$ 163.9 million per year [55].

Due to these tremendous high costs it must be possible of scientists, physicians, bioengineers and others to develop new techniques and new materials to reduce these high costs and to increase the improvement of patients.

But besides the high costs due catheter-related infections, the patients' longer hospital stays, and patients discomfort, mortality, and occasionally mortality are the most important problems which must be resolved. To reduce these complications it is necessary that the handling of the catheters must be done first after the numerous recommendation and guidelines available in the literature $[22,56,57]$.

Surface treatment of catheter is necessary, but of the inner and the outer surface. Therefore new technology must be developed for the surface treatment with antibitotics, antimicrobial substances and/or metal. New material and new-polymer-antibiotic systems are demanded. The developed of new biofilm to serve in a communication system termed quorum sensing [49], or molecules which inhibit quorum sensing signal generation among organism could block microbial biofilm formation [50]. This reqirement shows perhaps the new developed catheter material, the microdomain-structured surface [PURSMA-coated catheters, Gambro, Germany) [18]. Microdomain surfaces are considered the most biocompatible because they mimic the structure of natural biological surfaces. Microdomain structures are used to match the multiple requirements for improved catheter surfaces, that is reduced thromgenecity and improved antimicrobial properties. First results with these catheters are very encouraged.

Most important is the improvement of the handling of the catheters by the attending staff which is recommended in numerous available guidelines to reduce the tremendous high costs to treat the CRI and the discomfort and morbidity of the patients.

\section{Conclusion}

The results showed that catheters which were surface treated only on the outside had no advantages versus untreated catheters. In a retrospective study from 1992 to 2007, outer surface treated catheters with silver versus untreated catheters in 159 patients, who needed a large bore catheter, were investigated. There was no association between the in situ time and silver coated/uncoated catheters, resulting in the bacteriological examination, and patients' diagnosis or outcome. Reasons may be that on the surface treated catheters only the outer surface was coated with silver and the possibility of contamination by the handling during the extracorporeal treatments. Therefore, new materials and surface treatment on both surfaces, the inner and outer surface, are needed to save the tremendous high health costs for hemodialysis catheters, to reduce infection rates, and thrombus formations and to improve the patients' outcome.

\section{REFERENCES}

[1] L. Beloqui, S, Shaldon, L, Chiandussi and B. Higgs, "Haemodialysis by Percutaneous Catheterization of the Femoral Artery and Vein with Reginal Heparinisation," Lancet, Vol. 11, 1961, p. 857. 
[2] D. G. Maki, "Infection Due to Infusion Therapy," In: I. V. Bennett and P. S. Brachman, Eds., Hospitals Infections, Little Brown and Co., Boston, 1992, pp. 849-859.

[3] J. S. Groegor, A. B. Lucas and H. A. T. Thaler, "Infectious Morbidity Associated with Long-Term Use of Venous Access Devices in Patients with Cancer," Annals of Internal Medicine, Vol. 119, No. 12, 1993, pp. 1168-1174. doi:10.7326/0003-4819-119-12-199312150-00003

[4] R. Bambauer, P. Mestres and K. J. Pirrung, "Frequency, Therapy, and Prevention of Infections Associated with Large-Bore Catheter," ASAIO Journal, Vol. 38, No. 2, 1992, pp. 96-101.

[5] R. Bambauer, R. Inninger and K. J. Pirrung, "Complications and Side Effects Associated with Large-Bore Catheters in the Suclavian and Internal Veins," Artificial Organs, Vol. 18, No. 4, 1994, pp. 318-321. doi:10.1111/j.1525-1594.1994.tb02204.x

[6] C. M. Kjellstrand, G. G. Merino, S. M. Mauer, et al., "Complications of Percutaneous Femoral Vein Catheterization for Hemodialysis," Clinical Nephrology, Vol. 4, No. 1, 1975, pp. 37-40.

[7] A. Schwarzbeck, W. D. Brittinger, G. E. von Henning, et al., "Cannulation of Subclavian Vein for Hemodialysis Using Seldinger Technique," Transactions of American Society for Artificial Internal Organs, Vol. 27, No. 1, 1978, pp. 24-31.

[8] A. De Cubber, C. De Wolf, N. Lameire, et al., "Single Needle Hemodialysis with Double Headpump via the Subclavian Vein," Dialysis \& Transplantation, Vol. 7, No. 11, 1978, pp. 1261-1263.

[9] P. R. Uldall, P. R. Dyck and F. Woods, "A Subclavian Cannula for Temporary Vascular Access for Hemodialysis or Plasmapheresis," Dialysis \& Transplantation, Vol. 8, No. 9, 1979, pp. 963-968.

[10] C. T. Flynn and R. McGowan, "Subclavian Vein Catheter and Clockwork Pump," Dialysis \& Transplantation, Vol. 9, No. 5, 1980, pp. 556-616.

[11] R. Bambauer and G. A. Jutzler, "Jugularis-Interna Punktion zur Shaldon-Katheterisierung. Ein neuer Zugang für Akute Hämodialysen," Nieren-Hochdruckh, Vol. 9, No. 3, 1980, pp. 109-116

[12] R. Bambauer and G. A. Jutzler, "Transcutaneous Insertion of the Shaldon Catheter through the Internal Jugular Vein as Access for Acute Hemodialysis," Dialysis \& Transplantation, Vol. 11, No. 9, 1982, pp. 766-771.

[13] D. Philibert, M. Agharazii, E. Audy, et al., "Clinical Experience with a Chronic Hemodialysis Catheter with Symmetrical Tip Configuration (Palindrone TM)," JASN, Vol. 16, 2005.

[14] M. Allon, "Dialysis Catheter-Related Bacteremia Treatment and Prophylaxis," American Journal of Kidney Diseases, Vol. 44, No. 5, 2004, pp. 779-791.

[15] J. Nielsen, H. J. J. Kolosk and F. Espersen, "Staphylococcus aureus Bacteremia among Patients Undergoing Dialysis: Focus Dialysis Catheter-Related Cases," $\mathrm{Ne}$ phrology Dialysis Transplantation, Vol. 13, No. 1, 1988, pp. 139-145. doi:10.1093/ndt/13.1.139

[16] R. Locci, G. Peters and G. Pulverer, "Microbiological
Colonization of Prosthetic Devices. Microtopological Characteristics of Intravenous Catheters as Detected by Scanning Electron Microscopy," Zentralblatt für Bakteriologie, Mikrobiologie und Hygiene, Vol. 173, No. 2, 1981, pp. 285-292.

[17] J. W. Costerton, L. Montanaro and C. R. Arciola, "Biofilm in Implant Infections: Its Production and Regulation," International Journal of Artificial Organs, Vol. 28, No. 9, 2005, pp. 1065-1068.

[18] R. Bambauer, R. Schiel, S. Bambauer and P. Sioshansi, "Large Bore Catheters with Surface Treatments versus Untreated Catheters for Blood Access," The Journal of Vasclar Access, Vol. 2, No. 3, 2001, pp. 97-105.

[19] J. D. Maya, D. Carlton, E. Estrada, et al., "Treatment of Dialysis Catheter-Related Staphylococcus aureus Bacteremia with an Antibiotic Lock: A Quality Improvement Report," American Journal of Kidney Diseases, Vol. 50, No. 2, 2007, pp. 289-295. doi:10.1053/j.ajkd.2007.04.014

[20] G. K. Dogra, H. Herson, B. Hutchison, et al., "Prevention of Tunnelled Hemodialysis Catheter-Related Infections Using Catheter Restricted Filling with Gentamycin and Citrate: A Randomized Controlled Study," Journal of the American Society of Nephrology, Vol. 13, No. 8, 2002, pp. 2133-2139. doi:10.1097/01.ASN.0000022890.29656.22

[21] R. Bambauer, R. Schiel, P. Mestres and P. Sioshansi, "Scanning Electron Microscopic Investigation of Catheters for Blood Access," International Journal of Artificial Organs, Vol. 18, No. 5, 1995, pp. 326-331.

[22] R. Hollenbeck, V. Mickley, J. Brunkwall, et al., "Gefäßzugang zur Hämodialyse" Der Nephrologe, Vol. 4, No. 2, 2009, pp. 158-176. doi:10.1007/s11560-009-0281-0

[23] R. Bambauer, R. Schiel, S. Bambauer and P. Sioshansi, "Long-Term Catheters for Apheresis and Dialysis with Surface Treatment with Infection Resistance and Low Thrombogenicity," Therapeutic Apheresis and Dialysis, Vol. 7, No. 2, 2003, pp. 225-231. doi:10.1046/j.1526-0968.2003.00042.x

[24] R. Bambauer and G. A. Jutzler, "Lagekontrolle Großlumiger Zentraler Venenkatheter Mittels Intrakardialer Elektrographie," Intensivmed, Vol. 17, No. 6, 1980, pp. 1217.

[25] P. Sioshansi and E. J. Tobin, "Surface Treatment of Biomaterials by Ion-Beam Processes," Medical Plastics and Biomaterials, Vol. 2, 1995, pp. 50-59.

[26] P. Sioshansi, "New Process for Surface Treatment of Catheters," Artificial Organs, Vol. 18, No. 4, 1994, pp. 226271. doi:10.1111/j.1525-1594.1994.tb02193.x

[27] R. Bambauer, P. Mestres, R. Schiel, et al., "New Surface Treatment Technologies for Catheters Used for Extracorporeal Detoxication Methods," Dialysis \& Transplantation, Vol. 24, No. 5, 1995, pp. 228-237.

[28] P. Sioshansi, "Ion Implantation of Cobalt Chronicum Prosthetic Components to Reduce Polyethylene Wear," Orthopedics Today, Vol. 11, No. 1, 1991, pp. 24-25.

[29] D. Schierholz, A. Rump and W. Pulverer, "Klinische und Präklinische Effizienz Antimikrobieller Katheter," Anaesthesiol Intensivmed Notfallmed Schmerzther, Vol. 32, No. 5, 1997, pp. 289-305. doi:10.1055/s-2007-995058 
[30] R. Bambauer, R. Schiel, C. Bambauer and R. Latza, "Surface-Treted versus Untreated Large-Bore Catheters as Vascular Access in Hemodialysis and Apheresis," International Journal of Nephrology, Vol. 2012, 2012, Article ID: 956136,8 pages.

http://www.Hindawi.com/journals/ijn(2012/956136/

[31] R. Vanholder, N. Hoenich, S. Ringoir, et al., "Morbidity and Mortality of Central Venous Catheter Hemodialysis, a Review of 10 Years' Experience," Nephron, Vol. 47, No. 4, 1987, pp. 247-279. doi:10.1159/000184523

[32] G. Jean, B. Charra, C. Chazot, et al., "Risk Factor Analysis for Long-Term Tunnelled Dialysis Catheter-Related Bacteremias," Nephron, Vol. 91, No. 2, 2002, pp. 399405. doi:10.1159/000064279

[33] S. H. Kim, K. I. Song, J. W. Chang, et al., "Prevention of Uncuffed Hemodialysis Catheter-Related Bacteremia Using an Antibiotic Lock Technique: A Prospective, Randomised Clinical Trial," Kidney International, Vol. 69, No. 1, 2006, pp. 161-164. doi:10.1038/sj.ki.5000012

[34] E. A. King, D. McCoy, S. Desai, et al., "Vancomycin Resistant Enterococcal Bacteraemia and Daptomycin: Are Higher Doses Necessary?" Journal of Antimicrobial Chemotherapy, Vol. 66, No. 9, 2011, pp. 2112-2118. doi:10.1093/jac/dkr255

[35] I. Cui, A. Iwamoto and I. O. Lian, "Novel Mechanism of Antibiotic Resistance Originating in Vancomycin-Intermediate Staphylococcus aureus," Antimicrobial Agents and Chemotherapy, Vol. 50, No. 2, 2006, pp. 428-438. doi:10.1128/AAC.50.2.428-438.2006

[36] I. Cui, F. Tominage, H. M. Nenh, et al., "Correlation between Reduced Daptomycin Suscetibility and Vancomycin Resistance in Vancomycin-Intermediate Staphylococcus aureus," Antimicrobial Agents and Chemotherapy, Vol. 50, No. 3, 2006, pp. 1079-1082. doi:10.1128/AAC.50.3.1079-1082.2006

[37] D. G. Maki, J. K. Garman, J. M. Shapiro, et al., "An Attachable Silver-Impregnated Cuff for Prevention of Infection with Central Venous Catheters: A Prospective Randomized Multicenter Trial," The American Journal of Medicine, Vol. 85, No. 3, 1988, pp. 307-314. doi:10.1016/0002-9343(88)90579-7

[38] K. S. Tweden, J. D. Cameron, A. J. Razzouk, et al., "Silver Modification of Polyethylene Terphthalate Textile for Antimicrobial," ASAIO Journal, Vol. 43, No. 2, 1997, pp. M475-M481. doi:10.1097/00002480-199703000-00088

[39] A. Oloffs, C. Gosse-Siestrup, S. Bisson, et al., "Biocompatibility of Silver Coated Polyurethane Catheters and Silver Coated Dacron Material," Biomaterials, Vol. 15, No. 10, 1994, pp. 753-758. doi:10.1016/0142-9612(94)90028-0

[40] R. Bambauer, P. Mestres, R. Schiel, et al., "Surface Treated Large-Bore Catheters with Silver Based Coating Versus Untreated Catheters for Extracorporeal Detoxication Methods," ASAIO Journal, Vol. 44, No. 4, 1998, pp. 3003-3008. doi:10.1097/00002480-199807000-00013

[41] K. S. Rabindranath, T. Bansal, J. Adams, et al., "Systematic Review of Antimicrobials for Prevention of Hemodialysis Catheter-Related Infections," Nephrology Dialysis Transplantation, Vol. 24, No. 12, 2009, pp. 3763-3774. doi:10.1093/ndt/gfp327

[42] A. A. Hampton and R. J. Sheretz, "Vascular Access Infections in Hospitalised Patients," The Surgical Clinics of North America, Vol. 68, No. 1, 1988, pp. 57-71.

[43] C. Von Eif, W. Kohnen, K, Becker, et al., "Modern Strategies in the Prevention of Implant-Associated Infections," The International Journal of Artificial Organs, Vol. 28, No. 11, 2005, pp. 1146-1156.

[44] J. F. Timsit, O. Mimoz, B. Mourviller, et al., "Randomized Controlled Trial of Chlorhexidine Dressing and Highly Adhesive Dressing for Preventing Catheter-Related Infections in Critically Ill Adults," American Journal of Respiratory and Critical Care Medicine, Vol. 186, No. 12, 2012, pp. 1272-1278. doi:10.1164/rccm.201206-10380C

[45] L. Troidle and F. O. Finkelstein, "Catheter-Related Bacteremia in Hemodialysis Patients: The Role of the Central Venous Catheter in Prevention and Therapy," The International Journal of Artificial Organs, Vol. 31, No. 9, 2008, pp. 827-833.

[46] S. Cicalini, F. Palmieri and N. Petrosillo, "Clinical Review: New Technologies for Prevention of Intravascular Catheter-Related Infections," Critical Care, Vol. 8, 2004, pp. 157-162. doi:10.1186/cc2380

[47] G. Donelli, I. Francolini, A. Piozzi, et al., "New PolymerAntibiotic Systems to Inhibit Bacterial Biofilm Formation: A Suitable Approach to Prevent Central Venous CatheterAssociated Infections," Journal of Chemotherapy, Vol. 14, No. 5, 2002, pp. 501-507.

[48] S. Schmitt, G. Haase, E. Csomor, et al., "Inhibitor of Complement, Compstatin, Prevents Polymer-Mediated Mac-1 Up-Regulation of Human Neutrophils Independent of Biomaterial Type Tested," Journal of Biomedical Materials Research, Vol. 66A, No. 3, 2003, pp. 491-499. doi:10.1002/jbm.a.10031

[49] M. R. Parsek, D. L. Val, B. L. Hanzelka, et al., "Acyl Homoserine-Lactone Quorum-Sensing Signal Generation," Proceedings of the National Academy of Sciences of the United States of America, Vol. 96, No. 8, 1999, pp. 4360-4365. doi:10.1073/pnas.96.8.4360

[50] D. G. Davies, M. R. Parsek, J. P. Pearson, et al., "The Involvement of Cell-to-Cell Signals in the Development of a Bacterial Biofilm," Science, Vol. 280, No. 5361, 1998, pp. 295-298. doi:10.1126/science.280.5361.295

[51] H. J. Feldmann, S. Kobrina and A. Wasserstein, "Hemodialysis Vascular Morbidity," Journal of the American Society of Nephrology, Vol. 7, No. 4, 1996, 523- 535.

[52] S. Bambauer, "Cost Reduction Benefits of Applying an Antimicrobial Surface Treatment to Catheters," Personnel Communication, 1996.

[53] C. Schwebel, J. C. Lucet, A. Vesin, et al., "Economic Evaluation of Chlorhexidine-Impregnated Sponges for Preventing Catheter-Related Infection in Critically Ill Adults in the Dressing Study," Critical Care Medicine, Vol. 40, No. 1, 2012, pp. 11-17. doi:10.1097/CCM.0b013e31822f0604

[54] P. J. Pronovost, C. A. Goeschel, E. Colantuoni, et al., "Sustaining Reductions in Catheter-Related Bloodstream Infections in Michigan Intensive Care Units: Observa- 
tional Study," British Medical Journal, Vol. 340, 2010, p. c309. doi:10.1136/bmj.c309

[55] E. Toccanelli, G. Smith, A. Hiske, et al., "Epidemiology, Medical Outcomes and Costs of Catheter-Related Bloodstream Infections in Intensive Care Units of Four European Countries: Literature- and Registry-Based Estimates," Journal of Hospital Infection, Vol. 72, No. 2, 2009, pp. 97-103. doi:10.1016/j.jhin.2008.12.012

[56] N. P. O’Grady, M. Alexander, L. A. Burns, et al., "Gui- delines for the Preventions of Intravascular Catheter-Related Infections," Clinical Infectious Diseases, Vol. 52, No. 9, 2011, pp. e162-e193.

[57] T. Nagai, S. Kohsaka, T. Anzai, et al., "Low Incidence of Catheter-Related Complications in Patients with Advanced Pulmonary Arterial Hypertension Undergoing Continuous Epoprostenol Infusions," Chest, Vol. 141, No. 1, 2012, pp. 272-273. doi:10.1378/chest.11-1893 